\title{
A polarization filter for surface wave analysis: statistical approach
}

\author{
D. J. JIN (*)
}

Received on 9th March, 1979

\begin{abstract}
Polarization properties of seismic surface waves have been used for the identification and interpretation of small seismic events. In the present method of polarization filtering, generally elliptically polarizing noise is discriminated against specially polarizing surface waves. Statistically random nature of noise is taken into account in the determination of filter weights.
\end{abstract}

\section{RIASSUNTO}

Sono state usate le proprietà di polarizzazione delle onde sismiche superficiali per l'identificazione e l'interpretazione di piccoli eventi sismici. Nel metodo esposto in questo lavoro per il filtraggio della polarizzazione, il rumore, che generalmente si polarizza ellitticamente, è ben discriminato dalle onde superficiali polarizzate. Viene presa in considerazione, per la determinazione dei pesi del filtro, la natura statisticamente casuale del rumore.

(i) Geophysical Laboratory. Institute for the Earth and Man. Dallas. Texas. 


\section{INTRODUCTION}

Polarization filters have been developed and used in the analysis of small magnitude seismic events. In this paper polarization properties of surface wave relative to noise are described and statistical characteristics of noise are taken into account in designing filters to be used simultaneously for the analysis of seismic surface waves. Methods similar to the present one have been used by various authors (Shimshoni and Smith, 1964; Aki, 1964; Flinn, 1965; Griffin, 1966; Simons, 1968; Montalbetti and Kanasewich, 1970; Strauss, 1976). Kanasewich (1973) allocates a chapter of his text book for the discussion of polarization analysis. The difference of the present method from the representative methods used by previous investigators is briefly discussed in the following paragraphs. In this paper the medium through which surface waves propagate is assumed to be perfectly elastic and only fundamental modes of seismic surface waves are considered.

Shimshoni and Smith's method is for the discrimination of elliptically polarized noise against rectilinearly polarized body waves, whereas the present method of polarization filtering is for the discrimination of generally elliptically polarized noise against specially elliptically polarized surface waves. Noise is generally elliptically polarized since, due to its nature of randomness, the horizontal component may either lead or lag the vertical component by an arbitrary phase difference and the ellipticity which is the ratio of semiaxes is completely arbitrary and random. Rayleigh waves are specially elliptically polarized since the horizontal component may either lead or lag the vertical component, with the latter case being possible on a layered medium, by a certain phase difference of $\pi / 2$ and they are characterized by their ellipticity of about $2 / 3$.

The present method has an analogy to the method divised by Aki (1964) in the sense that the phase difference between components of surface waves is utilized, but they are quite different in their nature and objective. Aki uses the phase difference between Love and Rayleigh waves in order to determine 
the earthquake source phase difference in studying earthquake mechanism, while in this paper the phase difference between two components of Rayleigh waves is used in order to suppress the coexisting noise.

Flinn (1965) has utilized the rectilinearity and directionality of waves in order to separate compressional wave motion from the shear or surface wave motion, with emphasis on the isolation and identification of the $p P$ phase. Although the present method is philosophically same as that of Simons (1968), utilization of statistical properties of noise characteristics in designing the filters distiguishes the present method from that of Simons.

The following symbols are employed in the discussion which follows:

$-\vec{V}:$ vertical component of Rayleigh waves,

$-\vec{T}$ : transverse component of surface waves or Love waves,

$-\vec{R}$ : radial component of Rayleigh waves,

- V,T,R: amplitude of $\vec{V}, \vec{T}, \vec{R}$, respectively,

- $\varnothing_{V}, \varnothing_{T}, \varnothing_{R}:$ phases of $\vec{V}, \vec{T}, \vec{R}$, respectively,

- $V_{r}, T_{r}, R_{r}$ : real parts of $\vec{V}, \vec{T}, \vec{R}$, respectively,

- $V_{i}, T_{i}, R_{i}$ : imaginary parts of $\vec{V}, \vec{T}, \vec{R}$, respectively.

The seismogram from three-component seismometers gives:

$$
\begin{aligned}
& V \exp \left(i \varnothing_{V}\right)=V_{r}+i V_{i} \\
& T \exp \left(i \varnothing_{T}\right)=T_{r}+i T_{i} \\
& R \exp \left(i \varnothing_{R}\right)=R_{r}+i R_{i}
\end{aligned}
$$


where both polar and Cartesian coordinate representations are shown. We set a coordinate system such that:

$$
\begin{aligned}
& V \exp \left[i\left(\varnothing_{V}-\varnothing_{V}\right)\right]=V^{\prime}{ }_{r}=V \\
& T \exp \left[i\left(\varnothing_{T}-\varnothing_{V}\right)\right]=T_{r}+i T^{\prime}{ }_{i} \\
& R \exp \left[i\left(\varnothing_{R}-\varnothing_{V}\right)\right]=R^{\prime}{ }_{r}+i R^{\prime}{ }_{i}
\end{aligned}
$$

where $V_{r}^{\prime}, T_{r}^{\prime}, T^{\prime}{ }_{i}, R_{r}^{\prime}, R_{i}^{\prime}$, are the new coordinate versions of $V_{r}, T_{r}, T_{i}, R_{r}, R_{i}$ respectively. That is, by shifting the reference or origin time appropriately the vertical component of Rayleigh waves becomes purely real (see Figs $1 \mathrm{a}$ and $1 \mathrm{~b}$ ).

Since the real parts of Eq. [2] are of our utility, we shall consider only the real parts of Eq. [2]. Since $V, T^{\prime}{ }_{r}$, and $R^{\prime}{ }_{r}$ are all real quantities, they are in phase with each other. Therefore $T^{\prime}{ }_{r}$ and $R_{r}^{\prime}$ cannot, by definition, be radial component of Rayleigh waves.

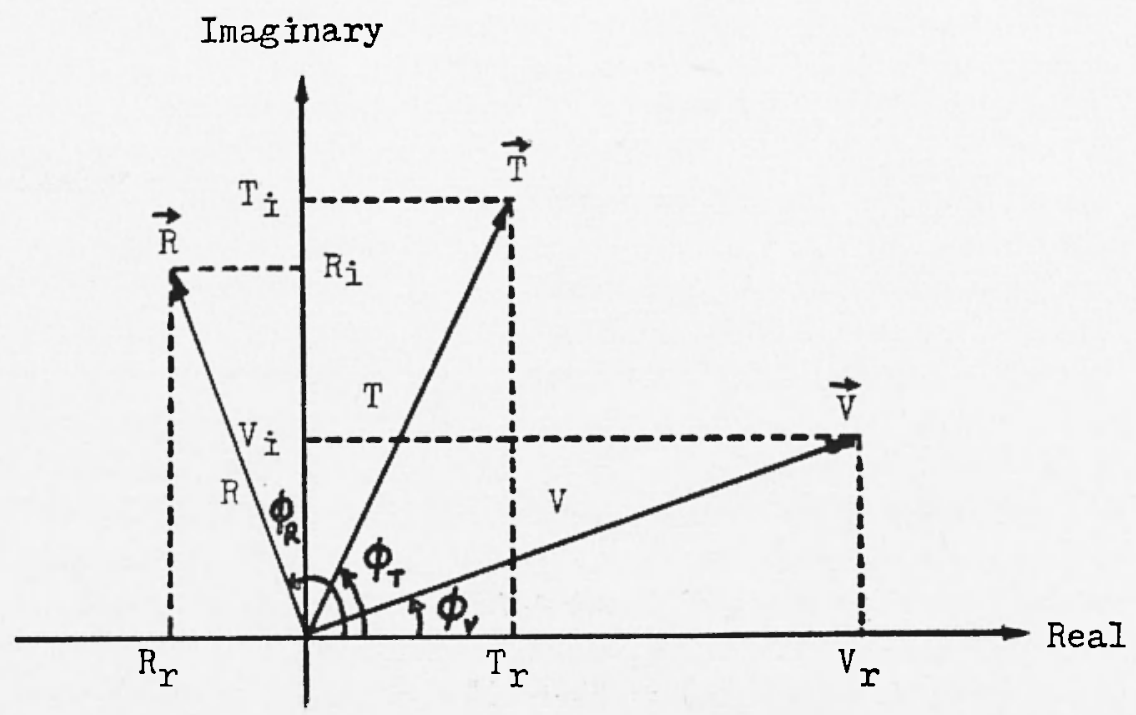

Fig. 1a - Three components of surface waves after rotation of coordinate axes, shown in the complex domain. 
After rotation of coordinate axes in the complex domain or correspondingly shift of reference or origin time in seismogram so that Eq. [2] holds, whole of radial component of Rayleigh waves should be purely imaginary so that it is out of phase with $V$ by $\pi / 2$ since $T_{r}^{\prime}$ and $R_{r}^{\prime}$ are, however, horizontal component of surface waves, but not radial component of Rayleigh waves, they should be Love waves whether they are desired ones or not.

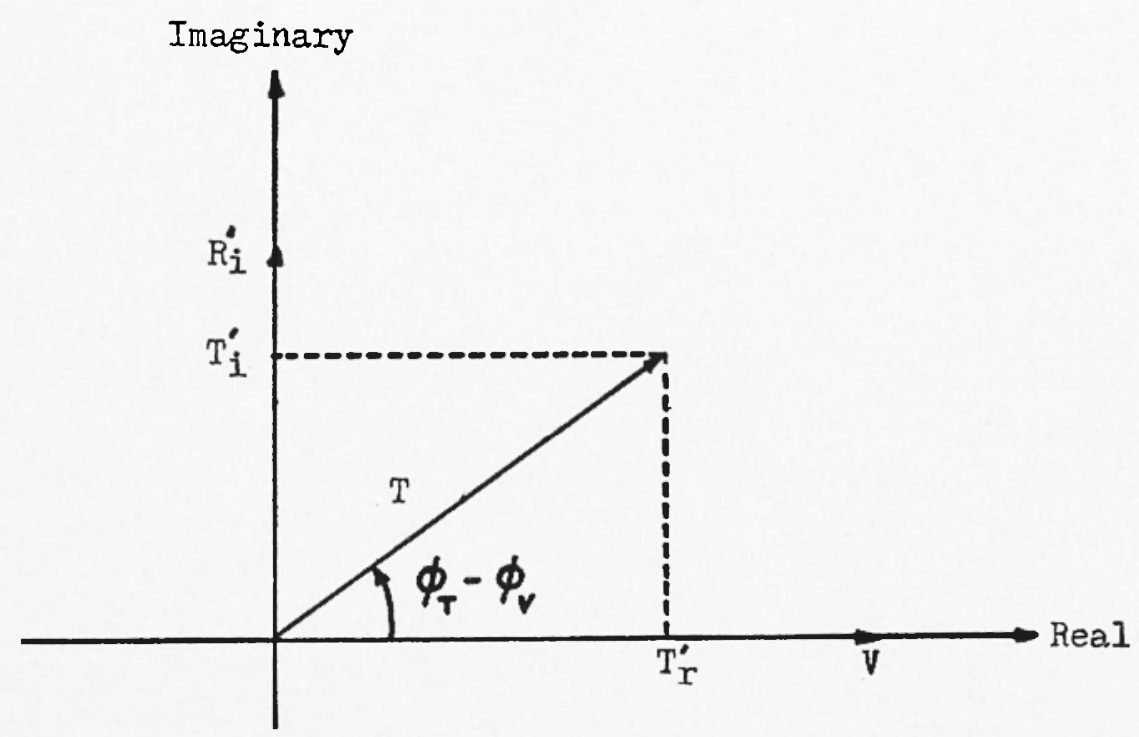

Fig. 1b - Three components of surface waves shown in the complex domain.

It is important to note that if there existed only pure signal of surface waves, $R_{r}^{\prime}$ would vanish as is shown in Fig. $1 \mathrm{~b}$. But due to ambient noise, $R_{r}^{\prime}$ is not usually zero. That is, we have not only the real part of desired Love waves but also part of noise which is observed through our seismometers as if it were part of desidered Love waves. The interpretation in the real words of what is said above is this: Due to the existing noise $R_{r}^{\prime}$, whose presence is observed in the Fourier component of the 
seismogram, the particle motion of Love waves has been deviated from the direction perpendicular to the radial direction. In other words, the propagation direction of Love waves has apparently been changed from its expected direction. Fig. 2 depicts this concept in a schematic manner.

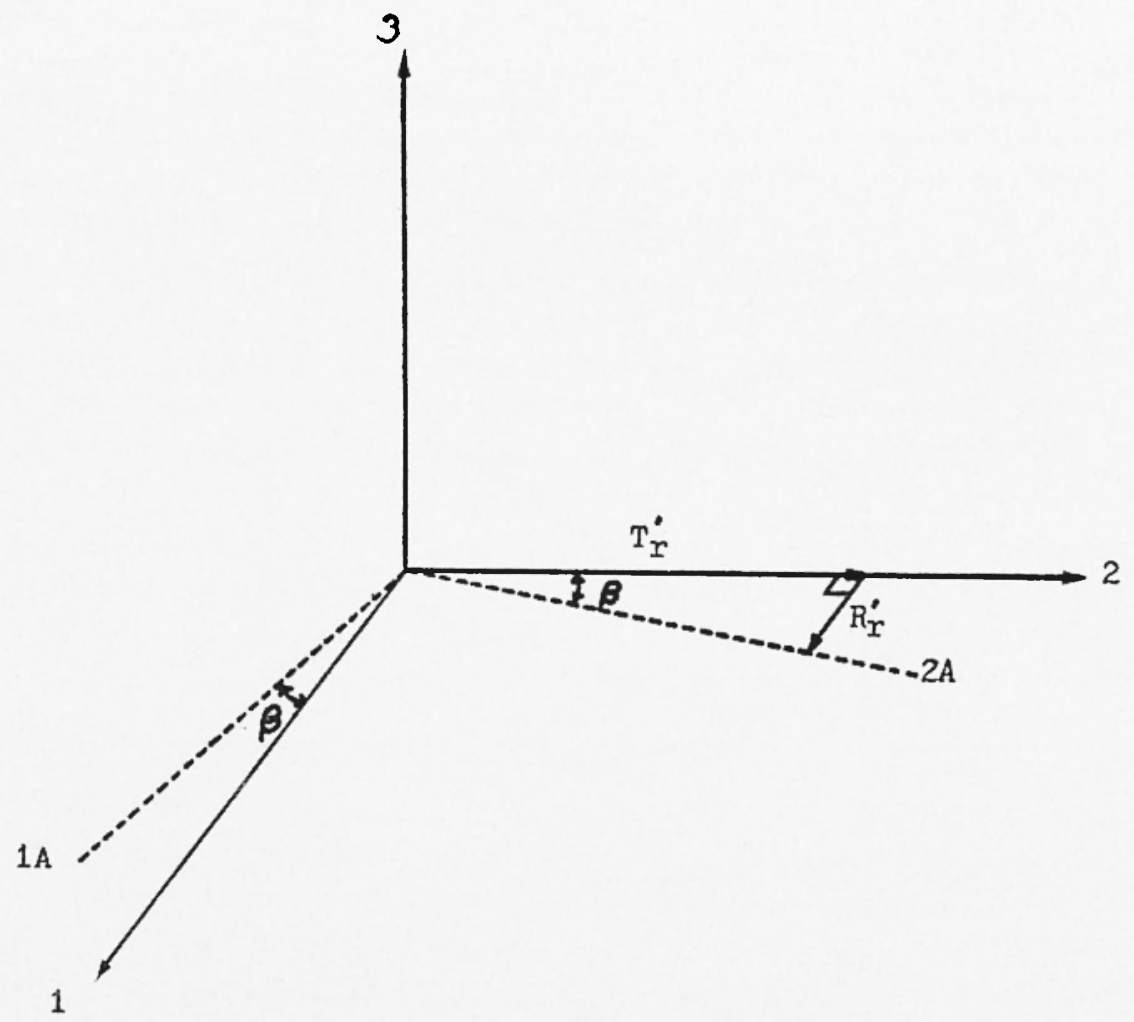

Fig. 2 - Components of the real part of Love waves shown in the real space domain; 1: Radial direction. 2: Transverse direction, which is the expected direction of the particle motion of Love waves. 3: Vertical direction.

1A: Love-wave propagation direction deviated from the radial direction due to noise.

2A: Love-wave particle-motion direction deviated from the transverse direction due to noise. 
From Fig. 2, the deviation angle $\beta$ of propagation direction of Love waves from its radial direction, can be computed by

$$
\beta=\arctan \left(R_{r}^{\prime} / T^{\prime}{ }_{r}\right)
$$

This angle $\beta$ is computed for each frequency component. We want to design a filter, by taking advantage of $\beta$, such that full weight would be assigned to the case where $\beta$ is zero or no noise exists and reduced or null weight as the noise increases. As it is discussed above, the present method utilizes the phase information from all three components of the surface waves. This is another aspect which makes the present method distinguished from that of Shimshoni and Smith, where no use of the transverse component was made (Shimshoni and Smith, 1964, p. 665). Strauss (1976) used similar method to the present one, but he tried to utilize an angle which corresponds to $\beta$ of this paper by shifting the observed transverse component back to the expected direction by rotating the axis by - $\beta$. In the present author's opinion, his method adds the observed noise to the actual transverse signal. The present method will give proper adjustment of the observed information according to the amount of the existing noise.

In designing a filter by making use of $\beta$, it should be borne in mind that the noise which yields the angle $\beta$ is inherently random in phase and consequently in its real part, which is the observed magnitude on a seismogram. Therefore an intelligent way in designing a filter which deals with something random may be to find out the statistical probability distribution of $\beta$ first. We will make reasonable assumptions on characteristics of the noise prior to the modeling of the distibution function for $\beta$.

Since $R^{\prime}$, discussed above is due to noise, we will adopt, simply or convenience, a new symbol $N_{R r}$ for $R_{r}^{\prime}$, by $N_{R r}$ implying the real part of noise in the radial direction. One point we postponed to discuss until this point is that as long as there exists the real part of noise in the radial direction, it is reasonable 
to assume the existence of the real part of the noise in the transverse direction also. Therefore $T^{\prime}{ }^{\prime}$ discussed above should be resolved into two components - the real part of desired Love waves, $\eta_{1}$, and the real part of the noise in that direction, $N_{\mathrm{Tr}}$. The breakdown of the observed Love waves is shown in Fig. 3. It is straightforward from Fig 3 to obtain the following:

$$
\beta=\arctan \left\{N_{R r} /\left(\eta+N_{T r}\right)\right\}
$$

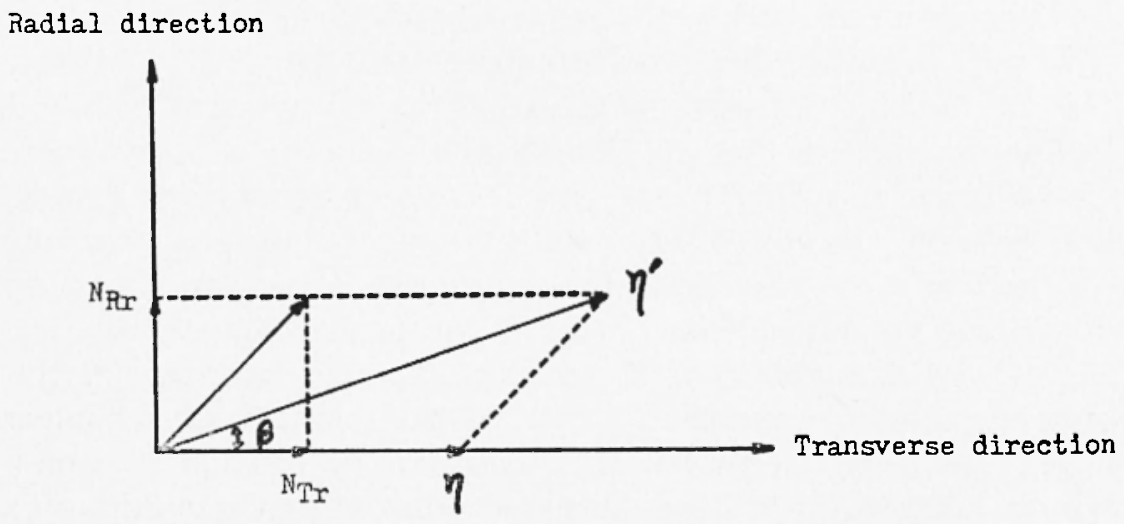

Fig. 3 - Breakdown of the real part of Love waves shown in the real space domain.

The two assumptions we want to make about the characteristics of the noise are as follows:

Assumption 1: The phase angles, $\Phi_{N R}, \Phi_{N T}$, and $\Phi_{N V}$, of the radial, transverse and vertical components of the noise are random and uncorrelated to each other.

Assumption 2: The amplitudes of the radial, transverse and vertical components of the noise are the same. From Assumption 2,

$$
\begin{aligned}
& N_{R r}=N_{R} \cos \Phi_{N R}=N \cos \Phi_{N R} \\
& N_{T r}=N_{T} \cos \Phi_{N T}=N \cos \Phi_{N T}
\end{aligned}
$$


where $N_{R}, N_{T}$ represent the amplitudes of the radial and transverse components of the noise and are identical to a quantity $N$. From Eqs. [4] and [5],

$$
\beta=\arctan \frac{\cos \Phi_{N R}}{\eta / N+\cos \Phi_{N T}}
$$

We assign a pair of random values ranging from zero to $2 \pi$ to $\Phi_{N R}$ and $\Phi_{N T}$ in Eq. [6] for a given signal-to-noise ratio, $\eta / N$, and obtain a corresponding value for $\beta$. By random in the above sentence we mean flat probability distribution of the values. We repeat this process sufficiently many times to warrant the statistical justification for the randomness, and to construct a probability distribution curve for $\beta$. Based on the probability distribution function for $\beta$, a filter for Love waves is designed.

Although the phase difference between the vertical and radial components of the Rayleigh waves should be $\pi / 2$, we expect that the noise would distort it to some extent, again requiring some form of filtering. We approach this problem with a statistical modeling similar to that for $\beta$ in Love waves. We wish to construct a probability distribution function for the apparent phase angle between the vertical and radial components of the Rayleigh waves under the influence of the noise. A summary of the symbols used in this part of the discussion and in Fig. 4 is as follows:

- $\zeta$ : vertical component of desired Rayleigh waves, and it is purely real after rotation of the coordinate axes,

$-\vec{N}_{V}$ : vertical component of noise;

- $N_{V}, \Phi_{N V}$ : amplitude and phase of $\vec{N}_{V}$

$-N_{V r}, N_{V_{t}}:$ real and imaginary parts of $\vec{N}_{\mathrm{V}}$,

- $\zeta^{\prime}$ : vertical component of apparent Rayleigh waves under the influence of the noise, 
$-\bar{\zeta}$ : radial component of desidered Rayleigh waves, and it is purely imaginary after rotation of the coordinate axes,

$-\vec{N}_{R}, N_{R}, \Phi_{N R}, N_{R r}, N_{R i}$ : radial component counter parts of $\vec{N}_{V}, N_{V}, \mathbb{D}_{N V}, N_{V r}, N_{V i}$, respectively,

- $\vec{c}^{\prime}$ : radial component of apparent Rayleigh waves under the influence of the noise, and has its phase different from that of $\xi$ by $\alpha$, and

- (D): the phase difference between the apparent vertical and radial components, i. e., $\zeta^{\prime}$ and $\xi^{\prime}$. of Rayleigh waves.

From. Fig. 4,

$$
\sin \gamma / N_{v}=\sin \left(\Phi_{N v}-\gamma\right) / \zeta
$$

Hence,

$$
\gamma=\arctan \frac{\sin \Phi_{N V}}{\bar{N}_{V}^{-}+\cos \Phi_{N V}}
$$

Similarly

$$
\sin \alpha / N_{R}=\sin \left(\pi / 2-\Phi_{N R}-\alpha\right) / \xi
$$

Hence,

$$
\alpha=\arctan \frac{\cos \Phi_{N R}}{\frac{\vec{\zeta}}{N_{R}}+\sin \Phi_{N R}}
$$

From the relation

$$
\Phi=\pi / 2-\alpha-\gamma
$$

and the two assumptions we made on the characteristics of the noise,

$$
\Phi=\pi / 2-\arctan \frac{\cos \Phi_{N R}}{\frac{(2 / 3) \zeta}{N}+\sin \Phi_{N R}}-\arctan \frac{\sin \Phi_{N V}}{\frac{\zeta}{N}+\cos \Phi_{N V}}
$$


In Eq. [9], we let $N_{V}=N_{R}=N$ and employed 2/3 for the ellipticity of the elliptic polarization of the Rayleigh waves without loosing generality. From Eq. [9], we construct a probability distribution curve for $\Phi$ in a similar manner to that for $\beta$, and based on this a filter for the Rayleigh waves is designed.

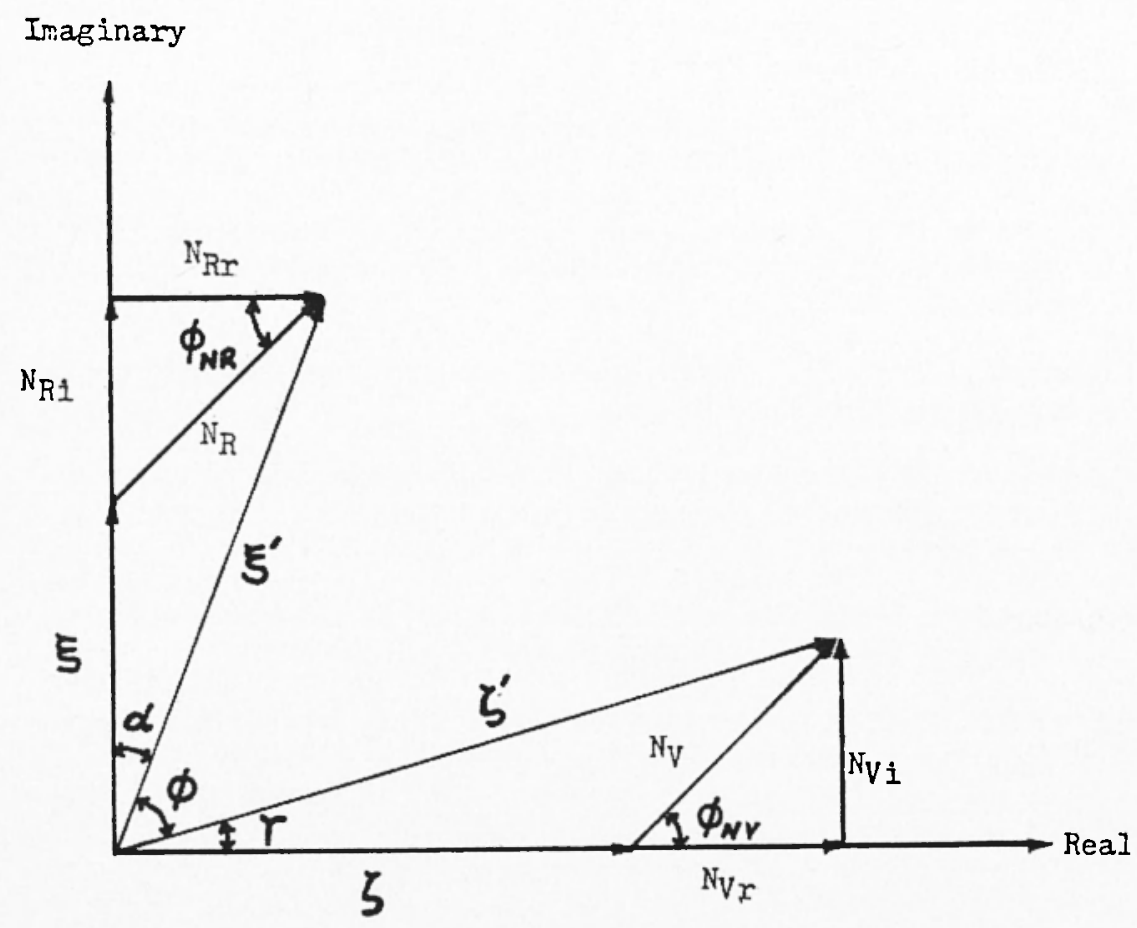

Fig. 4 - Apparent phase differences between the vertical and radial components of Rayleigh waves, shown in the complex domain.

\section{ACKNOWLEDGMENT}

The autor wishes to thank Dr. Stephen S. Lane of Texas Instruments, Inc. for his valuable suggestions and stimulating discussions with him on the subject of this paper. 


\section{REFERENCES}

AKI K., 1964. - Study of Love and Rayleigh waves from earthquakes with fault plane solutions or with known faulting. Part 1. A phase difference method based on a new model of earthquake source. "Bull. Seism. Soc. Am. ", 54, 2, pp. 511-527.

FLINN E.A., 1965. - Signal analysis using rectilinearity and direction of particle motion. "Proc. IEEE. ", 53, pp. 1874-1876.

GRIFFIN J.N., 1966. - Application and development of polarization (REMO$D E$ ) filters. "Seismic Data Laboratory Report 141 ". Teledyne Inc., Alexandria, Va.

Kanasl:wich E.R., 1973. - Time sequence analysis in geophysics. Univ. Alberta Press, Edmonton, Alberta.

Montalbetti J.F., Kanasewich E.R., 1973. - Enhancement of teleseismic body phases with a polarization filter. "Geophys. J. R. astr. Soc.", 21, pp. 119-129.

ShIMSHONi M., SMith S.W., 1964. - Seismic signal enhancenent with threecomponent detectors. "Geophysics ", 29, 5, pp. 664-671.

Simons R.S., 1968. - A surface wave particle motion discrimination process. “Bull. Seism. Soc. Am. ", 58, 2, pp. 629-637.

StRauss A.C., 1976. - Evaluation of an improved adaptive three-component processor. Texas Instruments, Inc., Alexandria, Va. 